\title{
Efficient Conditional Reliability Updating with Sequential Importance Sampling
}

\author{
Max Ehre ${ }^{1, *}$, Iason Papaioannou ${ }^{1}$, and Daniel Straub ${ }^{1}$ \\ 1 TU München, Risk Analysis Group, Theresienstrasse 90, 80333 München
}

\begin{abstract}
Data on the performance of engineering systems can and should be used to learn system parameters and update the system reliability estimate. This can be achieved in a Bayesian framework. In order to capture the dependence of the reliability estimate on the data used, we consider the probability of failure conditional on the updating variables, i.e. those variables on which data is available. Thus, the probability of failure becomes a random variable itself. In practice, the distribution of the conditional probability of failure may be obtained by solving a reliability problem at several samples from the updating variable space which causes a considerable increase in required computational effort. To address this challenge, we devise a procedure for the fast re-computation of failure probabilities within a sequential sampling method. We exploit the similarity of reliability problems with small distance in a suitable norm by sequentially initializing computations with failure samples of similar problem solutions.

Copyright line will be provided by the publisher
\end{abstract}

\section{Conditional probability of failure}

Consider a deterministic model $\mathcal{Y}$ with random vector input $\Theta$ that have joint distribution $\pi_{\Theta}$, i.e. $\mathcal{Y}: \Theta \rightarrow Y, \Theta \sim \pi_{\Theta}$. We define acceptable system behaviour in terms of the model output by using a limit-state function (LSF) $\tilde{g}(Y)$. By convention, $\tilde{g} \leq 0$ indicates system failure. Reliability analysis is concerned with estimating the probability of the failure event $F=\{\Theta$ : $g(\boldsymbol{\Theta}) \leq 0\}$, where $g=\tilde{g} \circ \mathcal{Y}$.

$$
\mathbb{P}(F)=\int_{\Omega_{\boldsymbol{\Theta}}} I(g \leq 0) \pi_{\boldsymbol{\Theta}}(\boldsymbol{\theta}) \mathrm{d} \boldsymbol{\theta}=\mathbb{E}_{\pi_{\boldsymbol{\Theta}}}[I(g \leq 0)], \quad I(g \leq 0)=\left\{\begin{array}{l}
1, g(\boldsymbol{\Theta}) \leq 0 \\
0, \text { else }
\end{array} .\right.
$$

While all components of $\Theta$ are random, one can typically distinguish reducible $\left(\Theta_{B}\right)$ and irreducible $\left(\Theta_{A}\right)$ uncertainty in the input variables, i.e. introduce a splitting $\boldsymbol{\Theta}=\left[\boldsymbol{\Theta}_{A}, \boldsymbol{\Theta}_{B}\right]^{T}$. Reducible uncertainties are subject to change through the incorporation of additional data, e.g. via Bayesian updating. The probability of failure conditional on the reducible inputs $\boldsymbol{\Theta}_{B}$ reads [3]

$$
P\left(\boldsymbol{\theta}_{B}\right)=\mathbb{P}\left(F \mid \boldsymbol{\Theta}_{B}=\boldsymbol{\theta}_{B}\right)=\int_{\Omega_{\boldsymbol{\Theta}_{A}}} I(g \leq 0) \pi_{\boldsymbol{\Theta}_{A} \mid \boldsymbol{\Theta}_{B}}\left(\boldsymbol{\theta}_{A} \mid \boldsymbol{\theta}_{B}\right) \mathrm{d} \boldsymbol{\theta}_{A}=\mathbb{E}_{\pi_{\boldsymbol{\Theta}_{A} \mid \boldsymbol{\Theta}_{B}}}\left[I(g \leq 0) \mid \boldsymbol{\Theta}_{B}\right]\left(\boldsymbol{\theta}_{B}\right) .
$$

That is, the probability of failure is a function of the outcome of the random vector $\Theta_{B}$ and thus a random variable itself. The distribution of $P\left(\boldsymbol{\theta}_{B}\right), \pi_{P}$, can be used to quantify the influence the data used for the Bayesian updating in $\boldsymbol{\Theta}_{B}$ has on the reliability analysis. To obtain a sampling estimate of $\pi_{P}$ based on $n_{B}$ realizations of $\boldsymbol{\theta}_{B}$, i.e. $\left\{\boldsymbol{\theta}_{B}^{j}, j=1, \ldots, n_{B}\right\}, n_{B}$ reliability problems over $\Omega_{\Theta_{A}}$ have to be solved:

$$
P\left(\boldsymbol{\theta}_{B}^{j}\right)=\int_{\Omega_{\boldsymbol{\Theta}_{A}}} I\left(g\left(\boldsymbol{\theta}_{A}, \boldsymbol{\theta}_{B}^{j}\right) \leq 0\right) \pi_{\boldsymbol{\Theta}_{A} \mid \boldsymbol{\Theta}_{B}}\left(\boldsymbol{\theta}_{A} \mid \boldsymbol{\theta}_{B}^{j}\right) \mathrm{d} \boldsymbol{\theta}_{A} .
$$

\section{Sequential Importance Sampling}

We use sequential importance sampling (SIS, [2]) to approximate integral (3) efficiently. SIS generates samples from a sequence of distributions $\left\{h_{i}(\boldsymbol{\Theta}), i=0, \ldots, M\right\}$ that gradually approach the optimal importance sampling density. The distribution sequence is constructed through introducing a smooth approximation of $I(g \leq 0)$ in the expression for the optimal importance sampling density. Once this optimal density is approached, the method estimates the probability of failure through importance sampling with the final importance sampling density $h_{M}$.

\section{Initializing SIS (SIS-I)}

The $I\left(g\left(\boldsymbol{\Theta}_{A}, \boldsymbol{\theta}_{B}^{j}\right) \leq 0\right)$ belong to a family of functions parameterized by $\boldsymbol{\theta}_{B}$, i.e. they may be similar in shape. The idea of this contribution is to initialize the SIS algorithm for solving the $j$-th reliability problem with samples from a previously solved reliability problem rather than $\pi_{\Theta_{A}}$. The goal is to select an initial distribution which is closest possible to the $j$-th optimal IS density. Two selection strategies are proposed, one of which has been used in the context of reliability-based optimization [1]:

\footnotetext{
* Corresponding author: max.ehre@tum.de.
} 


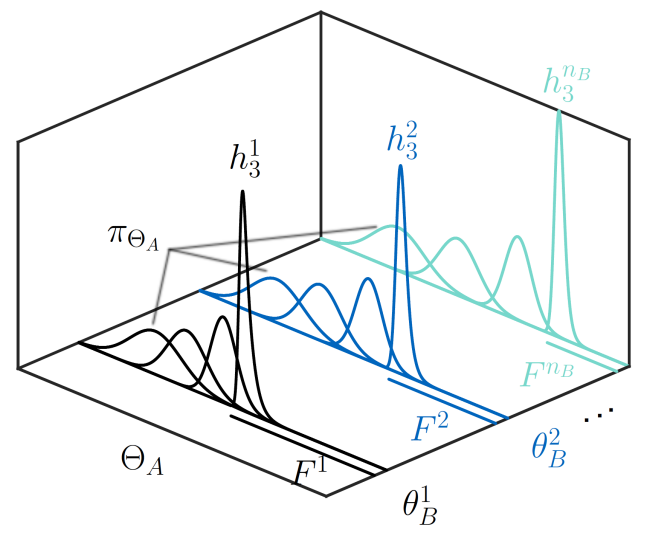

Fig. 1: Schematic SIS solution progress for $n_{B}$ reliability problems with LSFs $g^{j}=g\left(\boldsymbol{\Theta}_{A}, \boldsymbol{\theta}_{B}^{j}\right), j=1,2, \ldots, n_{B}$. (different colors).

1. Memoryless Approach The $\boldsymbol{\theta}_{B}^{j}$ are ordered by a $L_{2}$-nearest-neighbor search [4] on $\Omega_{\Theta_{B}}$. We then always pick $h_{0}^{j}=$ $h_{M-1}^{j-1}$.

2. Single-Point Search [1] We store all previously used densitites $h_{i}^{k}, k<j, i \leq M_{k}$ along with the locations of their maxima $\tilde{\boldsymbol{\theta}}_{i}^{k}=\underset{\boldsymbol{t} \in \Omega_{\boldsymbol{\Theta}}}{\arg \min } h_{i}^{k}(\boldsymbol{t})$. Then, we pick $i$ and $k$ s.t. $g^{j}\left(\tilde{\boldsymbol{\theta}}_{i}^{k}\right)$ becomes minimal under the constraint $g^{j}\left(\tilde{\boldsymbol{\theta}}_{i}^{k}\right) \geq 0$.

It is possible, that the selected density has little shared probability mass with the target density. In such case, the analysis becomes instable and is instead restarted from $\pi_{\Theta_{A}}$. In future work, this shall be adressed by moving samples from the selected density to regions of higher shared probability mass with the target via bridging rather than restarting.

\section{Numerical Example}

We are testing the approach (SIS-I) with a two-dimensional truss example, which is described in [5]. For the Bayesian updating, we assume measurement noise $\epsilon \sim \mathcal{N}\left(0, \sigma_{\epsilon}\right)$ and generate a measurement via $\mathcal{D}=\mathcal{Y}\left(\boldsymbol{\mu}_{\Theta_{A}}, \boldsymbol{\theta}_{B}^{\text {true }}\right)+2 \epsilon$. The likelihood and LSF definitions read

$$
\mathcal{L}\left(\boldsymbol{\theta}_{B}\right) \sim \exp \left\{-\frac{1}{2}\left(\frac{\mathcal{Y}\left(\boldsymbol{\mu}_{\boldsymbol{\Theta}_{A}}, \boldsymbol{\theta}_{B}\right)-\mathcal{D}}{\sigma_{\epsilon}}\right)^{2}\right\}, \quad g=\mathcal{Y}(\boldsymbol{\Theta})-Y_{c r i t}, \quad Y_{c r i t}=\mathcal{Y}\left(1.5 \boldsymbol{\mu}_{\boldsymbol{\Theta}_{A}}, \boldsymbol{\mu}_{\boldsymbol{\Theta}_{B}}\right) .
$$

Reference solutions are computed via performing SIS from $\pi_{\Theta_{A}}$ at each sample.
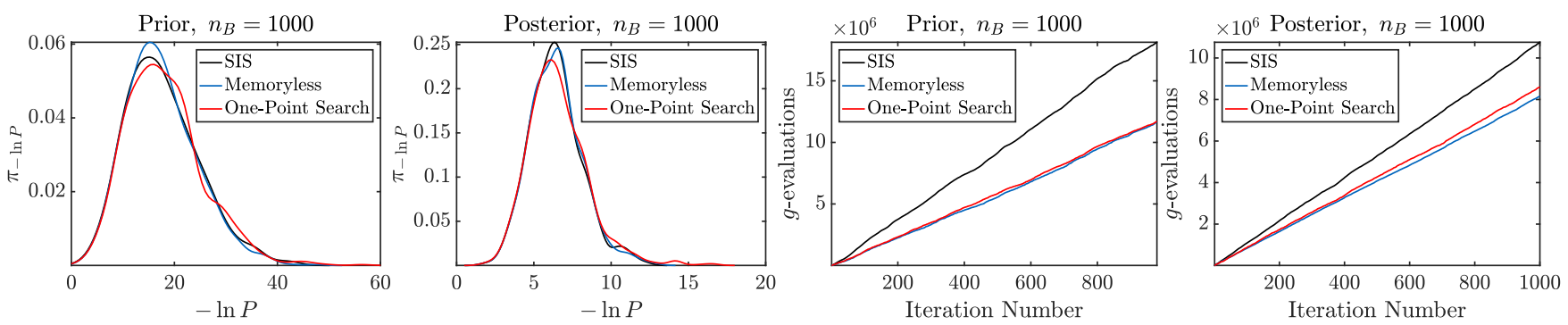

Fig. 2: Prior \& Posterior $\ln P$-densities and respective accumulated number of limit-state function evaluations.

\section{Conclusions}

We proposed an initialization scheme for solving a sequence of reliability problems with SIS which yields a reduction in computational cost by approximately $15 \%$ (posterior) to $30 \%$ (prior) at good accuracy. The computational gains are expected to increase significantly with replacing the analysis restarting by a bridging scheme.

\section{References}

[1] Beaurepaire P., Jensen H.A., Schuëller G.I. and Valdebenito M.A., Probabilistic Engineering Mechanics 34, 48 - 67 (2013).

[2] Papaioannou I., Papadimitriou C. and Straub D., Structural Safety 62, 66 - 75 (2016).

[3] Der Kiureghian, A. and Ditlevsen, O., Structural Safety 31, 105 - 112 (2009).

[4] Friedman, J. H., Bentely J. and Finkel, R. A., ACM Transactions on Mathematical Software 3, 209-226 (1977).

[5] Lee S.H. and Kwak B.M., Journal of Computational Physics 28, 261-272 (2006). 